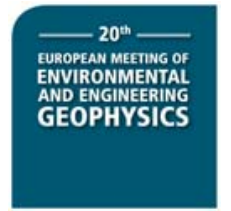

\title{
Introduction
}

This study presents a new electrical resistivity model for the near surface geology of Great Britain. It describes the first known national model of electrical resistivity at 1:50k scale. Due to the paucity of measured in situ electrical resistivity data a modeling scheme is adopted to attribute the spatial compilation. The British Geological Survey's (BGS) National Geotechnical Database (Self, 2012) is utilized to derive synthetic modeled resistivity distributions for all of the lithostratigraphic units described in the near surface geological model of Great Britain. Resistivity values are calculated using an effective medium algorithm (Berg, 2007) and the central moments of the statistical distributions are used to populate the map. Alongside this primary output, the expected range of resistivity is derived from the difference between the $20^{\text {th }}$ and the $80^{\text {th }}$ percentiles of the distributions.

\section{Geology of Great Britain}

The near surface geology of England, Scotland and Wales is described in the soil-parent material map of Great Britain (Lawley, 2008). This model characterizes the geological unit beneath the agricultural soil and is derived from the 1:50k scale bedrock and superficial geological maps of Great Britain, known as DiGMapGB-50 (British Geological Survey, 2011), see Figure 1.

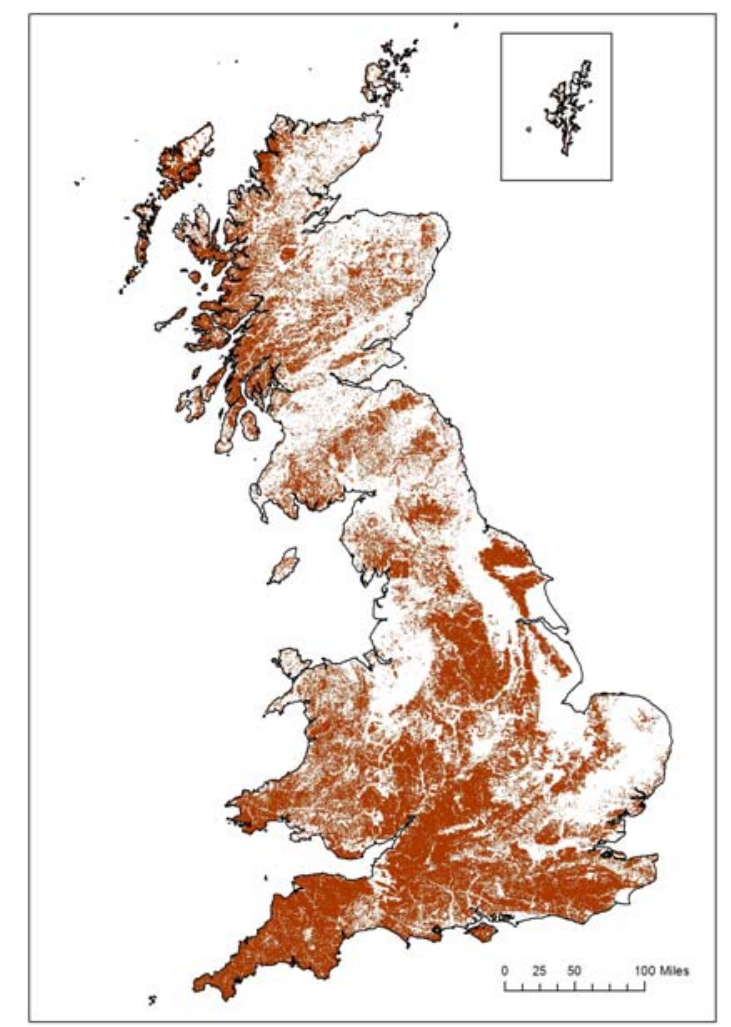

Figure 1. The distribution of bedrock (brown) and superficial (white) units in the near surface geological model of Great Britain.

A rigorous lithostratigraphic attribution scheme which differentiates the subsurface on the basis of both the specific deposit and its lithology is used in this study and the geological variability is illustrated through over 1.4 million polygons representing 9514 unique classifications.

\section{Method}

Electrically conductive clay minerals in porous formations make a significant contribution to the overall resistivity. Schemes have been developed which include the conductivity of clays (Simandoux, 1963, Waxman and Smits, 1968) in the calculation of bulk resistivity. Berg (2007) proposed an effective medium approach to determine the whole rock resistivity from geotechnical 
properties. The calculation requires, as inputs, the porosity, saturation, clay content, pore fluid resistivity and clay grain resistivity (for lithologies with a clay component). For each geological classification these variables are likely to cover a significant range. As such, minimum and a maximum values, for each parameter, are defined for every unit. Resistivities are derived by randomly selecting the value of each variable from within its range. 5000 different realizations of resistivity are calculated for each geological classification to generate a synthetic, modeled, distribution. From this distribution the central moment is extracted along with the $20^{\text {th }}$ and $80^{\text {th }}$ percentile values. These percentiles best summarize the expected range of resistivity.

The effective medium algorithm enables the formation water conductivity and the conductivities of any disperse constituents (and their geometric factors) to be accurately combined. The method accommodates the associated grain conductivities of clays into the resistivity calculation by considering each rock type as a saturated clay-sand of variable composition. The method requires the repeated solution of the Hanai-Bruggeman equation (Bussian, 1983) which determines the conductivity of a two component mixture of matrix and water,

$\Phi=\left(\frac{\sigma_{x x}}{\sigma_{g}}\right)^{\frac{m-2}{m}}\left(\frac{\sigma_{g}-\varepsilon_{x}}{\sigma_{w s}-\omega_{\gamma}}\right)$,

where $\sigma_{\mathrm{w}}=$ water conductivity, $\sigma_{0}=$ mixture conductivity, $\sigma_{\mathrm{r}}=$ matrix conductivity, $\mathrm{m}=$ cementation exponent and $\phi=$ porosity and the electrical conductivity, $\sigma$, is the reciprocal of resistivity, $\rho$.

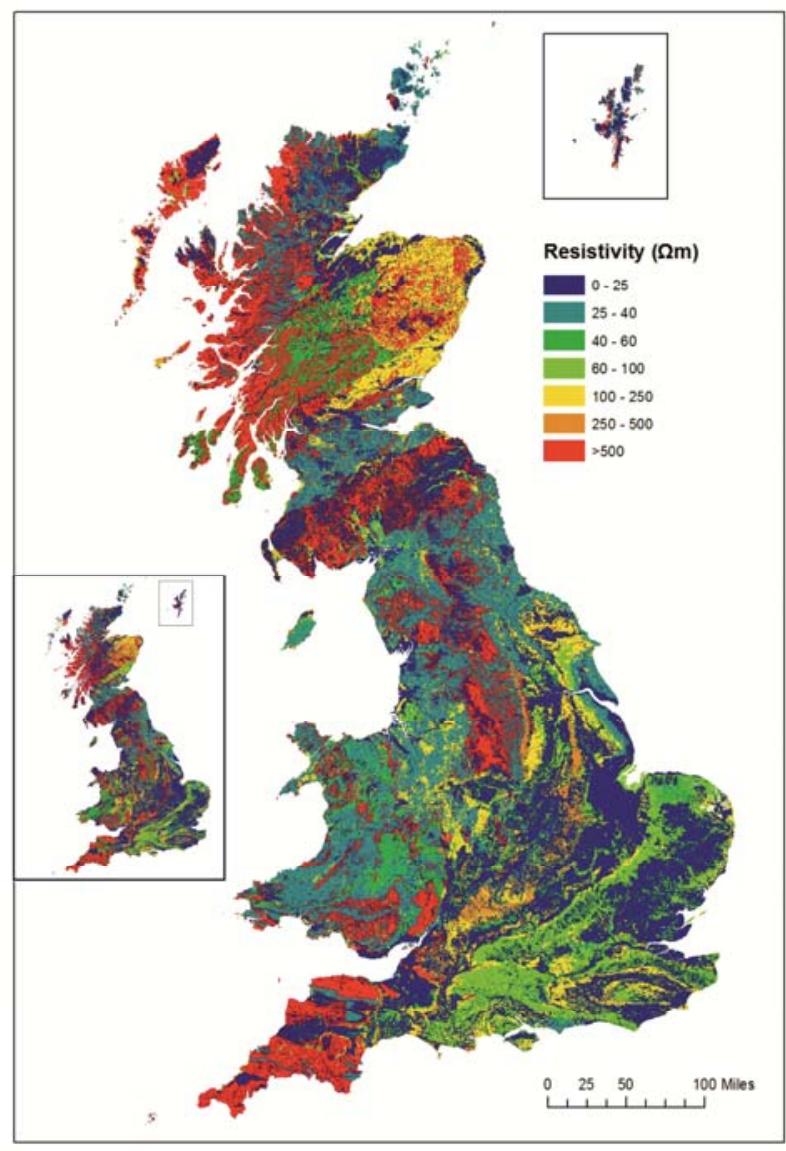

Figure 2. The near surface electrical resistivity map of Great Britain and, inset, the expected range of electrical resistivity (between the 20th and 80th percentiles) from modeled data.

The derived resistivity values are coupled to the soil-parent material map of Great Britain (Lawley, 2008). This results in a spatial distribution of resistivity across the extent of the map compilation. 
The central moment of the statistical resistivity distribution is displayed in Figure 2 alongside a map highlighting the modeled resistivity range.

\section{Comparison with existing data}

Prior to the bedrock conductivity model of the UK produced in 2013 (Beamish, 2013) the previous national compilation of electrical resistivity was shown to the Physical Society in 1935 (Smith-Rose, 1935). Beamish (2013) used a lithological classification of bedrock at a scale of 1:625k and his analysis comprised just 86 lithology categories from the bedrock geological lexicon of the UK. Specific lithologies were joined to a table of apparent half space conductivity values derived from airborne electromagnetic (AEM) surveys acquired over the UK (Beamish and White, 2012). Figure 3 shows a comparison of the two methods after the electrical resistivity model of the near surface geology of Great Britain has been clipped to locations where bedrock is mapped in the soil-parent material map. This allows a comparison between the two methodologies.

There is a general agreement between the two models, generated with dissimilar approaches. The north of Scotland, the Scotland and England border regions and the south-west of England exhibit high resistivity in both compilations whilst the south and east of England, where younger more porous geological units occur, are more conductive. The near surface map is clearly more resistive, especially in the north and west. This is expected since the models have different depths of interest with a greater variability in the saturation and porosity expected close to the surface. In some regions significant differences are apparent. These highlight the advantages of a lithostratigraphic, rather than a basic lithological, classification of geology. For example, resistivity values of the Torridon sandstones in the north west of Scotland, highlighted by the black oval in Figure 3, show considerable disparity.
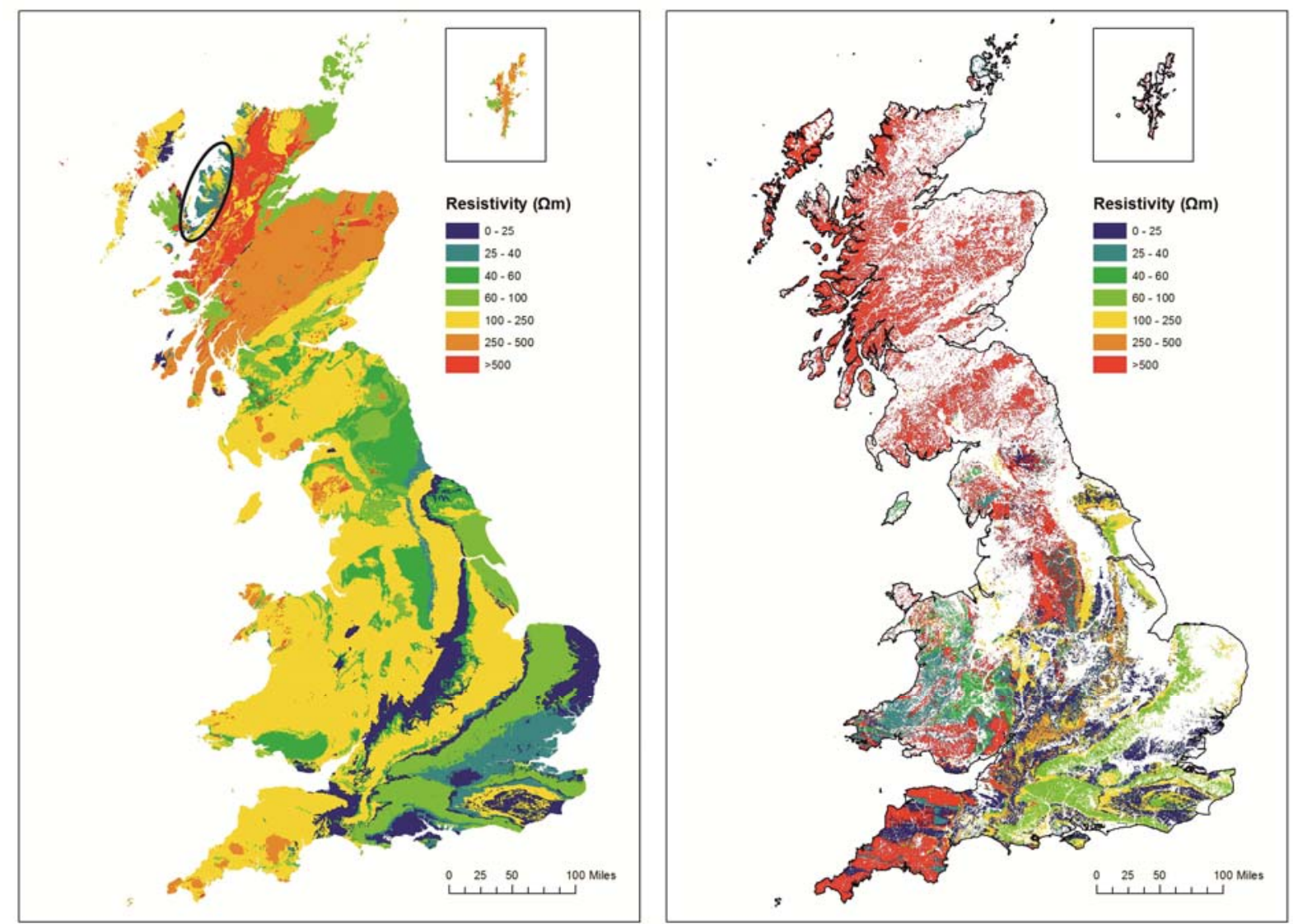

Figure 3. Comparison between bedrock resistivity models. (Left) the 1:625k lithologically classified distribution (Beamish, 2013); and (right) the 1:50k lithostratigraphically classified distribution. 


\section{Conclusions}

Modeled resistivity distributions have been derived using an effective medium algorithm for each of the lithostrigraphic units classified in the near surface geological model of Great Britain. This methodology utilizes geotechnical data which had been collected for other purposes. The resulting map compilation presents the electrical resistivity of the top 3 to $5 \mathrm{~m}$ of geology (beneath the base of pedological soil) across England, Scotland and Wales. The scheme generates a map compilation of modeled resistivity at $1: 50 \mathrm{k}$ scale. Adopting a rigorous geological attribution scheme which differentiated the subsurface on the basis of the geological unit as well as the rock classification (lithology) has ensured the variability in electrical resistivity is best captured across the spatial extent of the map.

In the broadest sense, the resulting map shows a steady increase in electrical resistivity from SE England to NW Scotland. This general trend is expected since the main lithological controls on resistivity are porosity and clay content. Generally, the bedrock of Great Britain becomes older from SE to NW. Overlying this general trend, the influence of superficial deposits, which usually display lower resistivity, is clear. The primary output of this study reports the central moment of the modeled resistivity distribution alongside a measure of the range. This data set is expected to be particularly relevant to the major power distribution networks that require resistivity data to design earthing installations. The methodology developed in this study can be applied to other regions where suitable geotechnical information is held.

\section{Acknowledgements}

David Beamish is thanked for access to his 1:625k scale UK conductivity map. This paper is published with the permission of the Executive Director, British Geological Survey.

\section{References}

Beamish, D. [2013] The bedrock electrical conductivity map of the UK. Journal of Applied Geophysics, 96, 87-97.

Beamish, D and White, J.C. [2012] Mapping and predicting electrical conductivity variations across southern England using airborne electromagnetic data. Quarterly Journal of Engineering Geology and Hydrogeology, 45, 99-110.

Berg, C. [2007] An effective medium algorithm for calculating water saturations at any salinity or frequency. Geophysics, 72, E59-E67.

British Geological Survey [2011] Digital Geological Map of Great Britain 1:50 000 scale (DiGMapGB-50) data [CD-Rom]. Version 6.20. Keyworth, Nottingham: British Geological Survey.

Bussian, A.E. [1983] Electrical conductance in a porous medium. Geophysics, 48, 1258-1268.

Lawley, R. [2008] The soil-parent material database: a user guide, British Geological Survey Open report, OR/08/034.

Self, S., Entwisle, D.C. and Northmore, K. [2012] The structure and operation of the BGS National Geotechnical Properties Database. Version 2. Nottingham, UK, British Geological Survey, 68pp. (IR/12/056).

Smith-Rose, R.L. [1935] The electrical properties of soil at frequencies up to 100 Megacycles per second; with a note on the resistivity of the ground in the united kingdom. Proceedings of the Physical Society, 47, 923-931. 